Research Journal of Applied Sciences, Engineering and Technology 16(4): 160-165, 2019

DOI: $10.19026 /$ rjaset. 16.6020

ISSN: 2040-7459; e-ISSN: 2040-7467

(C) 2019 Maxwell Scientific Publication Corp.

Submitted: February 12, 2019

Accepted: May 23, 2019

Published: September 15, 2019

\title{
Research Article \\ Revision of Request to Send (RTS) Threshold to Improve Efficiency of IEEE 802.11n Wireless Ad hoc Networks
}

\author{
${ }^{1}$ Mehdi Effatparvar, ${ }^{1,2}$ Mohammadreza Effatparvar, ${ }^{1,2}$ Peyman Teymoori and ${ }^{1,2}$ Naser Yazdani \\ ${ }^{1}$ Computer Department, Ardabil Branch, Islamic Azad University, Ardabil, Iran \\ ${ }^{2}$ ECE Department, University of Tehran, Tehran, Iran
}

\begin{abstract}
In this study, we show that high-speed IEEE 802.11n wireless ad hoc networks suffer from loss of efficiency at network layer. Through extensive simulations, we find the problems of unfairness among traffic flows, packet drops, increased delay and decreased throughput in these networks. We believe the root cause of these problems is RTS Threshold implying that setting an appropriate threshold value can solve most of the problems. To the best of our knowledge, this is the first work that finds this problem and to solve it, we propose an analytical model by which the RTS threshold is set. The simulation shows that this setting can improve the efficiency of IEEE $802.11 \mathrm{n}$ networks up to $25 \%$ in terms of throughput.
\end{abstract}

Keywords: Ad hoc networks, efficiency, IEEE 802.11 n, routing, threshold

\section{INTRODUCTION}

To support rich multimedia applications such as High-Definition Television (HDTV) and DVDs, IEEE $802.11 \mathrm{n}$ provides physical rates of up to $600 \mathrm{Mbps}$ (Wang and Wei, 2009). It also defines two modes of operation: Distributed Coordination Function (DCF) which is ad hoc and based on CSMA/CA and Point Coordination Function (PCF) which is centralized. An ad hoc network is a dynamically reconfigurable wireless network with no fixed wired infrastructure. Due to its error-prone and dynamic nature, several proposals have been presented for routing.

Due to significance of routing in ad hoc networks, in this study, we investigate efficiency of the network layer of high-speed wireless ad hoc networks. Since IEEE $802.11 \mathrm{n}$ promises higher data rates at physical layer, it is important to investigate if it is possible to get the same proportional efficiency at higher layers. Some works (Wang and Wei, 2009; Li et al., 2009; Lin and Wong, 2006) show efficiency of the protocol at the MAC layer, but its effects on the routing efficiency are still unknown.

In Banchs and Vollero (2006) an analytical model for IEEE 802.11e EDCA function has been presented. In (Bianchi, 2000) authors presented a model for the throughput of IEEE 802.11 single-hop links under ideal channel conditions and saturated traffic, i.e., each node always has a frame available for transmission.
Frohn et al. (2010) characterize the effective throughput for multi-hop paths in IEEE $802.11 \mathrm{n}$ based wireless mesh networks has been proposed. We can find the analyzing the performance of IEEE 802.11n over single-hop links in a testbed in Shrivastava et al. (2008). Karlsson et al. (2009) reported on the performance of TCP in the presence of packet aggregation. Opposed to Karlsson et al. (2009) and Shrivastava et al. (2008), provide an analytical model considering the frame aggregation capabilities of IEEE 802.11n.

\section{LITERATURE REVIEW}

Kim et al. (2008) proposed a modification of the IEEE 802.11 MAC to allow aggregation of unicast and broadcast frames. Acharya et al. (2008) proposed WOOF, an optimized rate adaption scheme for WLAN. As one of the well-known routing protocols, we chose AODV (Perkins and Royer, 1999) although due to similar characteristics, our results are valid for most of the on-demand approaches. Through extensive simulations, we show that due to high control overheads, high-speed wireless ad hoc networks suffer from a problem that we call the RTS threshold problem. It means that RTS threshold not only depends on packet transmission time, but it also depends on other factors. Furthermore, there is a trade off in using RTS/CTS frames due to their overheads. We solve this problem using our proposed method, RAS-RTS. 
In Effatparvar et al. (2016) and Effatparvar et al. (2017) authors proposed the application of IEEE 802.11 in wireless body area sensor network. Also by using body area sensor network a new thermal aware routing protocol has been proposed (Bahrami and Effatparvar, 2017).

There are many papers have been proposed based on optimization by Effatparvar and Garshasbi (2014), Molaiy and Effatparvar (2014) and Garshasbi and Effatparvar (2013). Effatparvar et al. (2007) presented new distributed method to reducing the energy for the communication between nods and coordinator. Also Yoder et al. (2015), Fazelpour et al. (2016) and Shankar et al. (2015) used the optimization methods in Meso-Scaled material. Saffar Ardabili et al. (2007) evaluated efficiency score of Decision Making Units (DMUs) by the undesirable outputs. Aghayi et al. (2016) measured efficiency measure using common set of weights in present of uncertainty based on robust optimization. Aghayi (2017) proposed the approach to obtain cost efficiency of DMUs by fuzzy data. Aghayi and Maleki (2016) measured the efficiency of bank branches of Ardabil, Iran using robust optimization theory and undesirable outputs. Rostamy and Aghayi (2011) suggested the method for calculating overall profit efficiency using uncertainty as fuzzy in data. Aghayi and Ghelejbeigi (2016) presented the improvement of cost efficiency based on resource allocation. Aghayi (2016) computed revenue efficiency of DMUs with undesirable and fuzzy data. Salehpour and Aghayi (2015) calculated the most revenue efficiency with price uncertainty.

\section{MATERIALS AND METHODS}

IEEE $802.11 \mathrm{n}$ DCF supports two modes of handshake: Basic Access and RTS/CTS Access. In Basic access, nodes just send frames and wait to receive acknowledgement. RTS/CTS control frames are used in RTS/CTS access to reserve the channel and solve the hidden node problem.

Studies such as Wang and Wei (2009) and Li et al. (2009) assume a single-hop network while controls packets, RTS/CTS, are disabled. Reference Wang and Wei (2009) proposes that RTS/CTS handshake should be disabled due to its overhead on the MAC layer and Tinnirello et al. (2005) calculates the optimal RTS threshold only by considering packet transmission time. Since an ad hoc network is usually a multi-hop network, disabling RTS/CTS raises problems such as hidden and exposed nodes. Therefore, just disabling RTS/CTS handshake does not sound reasonable without a careful study.

In order to investigate performance at the network layer, we run extensive simulations in NS-2 (http://www.isi.edu/nsnam/ns/). We modified an implementation of IEEE $802.11 \mathrm{n}$ proposed by (Wang

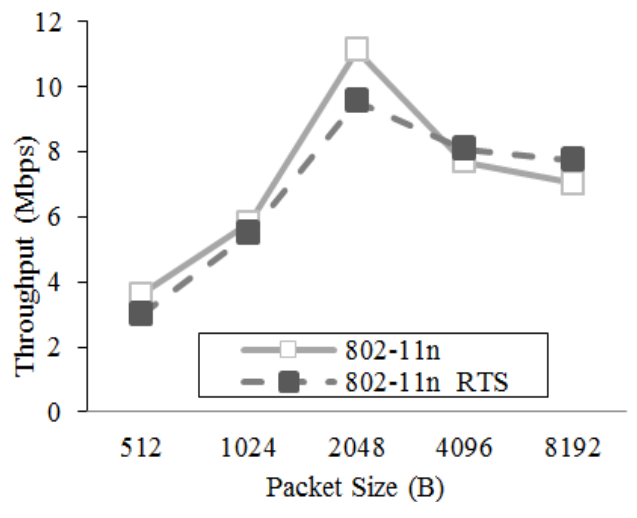

Fig. 1: Throughput for various packet sizes

and Wei, 2009) to make it routing-aware since it was used only in a single-hop scenario.

In our simulations, there are 50 nodes uniformly distributed in a $1000 * 1000 \mathrm{~m}^{2}$ environment. Nodes move $1 \mathrm{~m} / \mathrm{sec}$, with a pause time of $10 \mathrm{sec}$ and use the random walk model. Transmission range, data and basic rates, MAC queue size, maximum aggregation size, ACK type and RTS threshold are $225 \mathrm{~m}, 54$ and $8 \mathrm{Mbps}, 70,40000$ bytes, BlockACK and 311 bytes, respectively.

We assumed a large maximum aggregation size to increase the MAC layer efficiency even though the actual aggregation size varies during simulation depending on the selected packet size. There are 15 data flows, each generating 200 UDP packets per second, using the AODV routing protocol. The simulation ran for $300 \mathrm{sec}$.

We assumed a small RTS threshold to enable RTS/CTS access for all transmissions. In the following figures, Basic access and RTS/CTS access are shown by "802.11n" and "802.11n_RTS", respectively. Results are averaged over 10 different runs, with $95 \%$ confidence interval lower than 0.02 .

Figure 1 shows operational throughputs of the two modes. Throughput is defined as the total number of transmitted bits over the simulation time. Smaller packets result in smaller MAC aggregated data frames and increasing the size improves throughput. Sizes larger that $2 \mathrm{~KB}$ decrease throughput due to increase in transmission times and consequently more buffer overflow and packet drops. In addition, large packets decrease throughput of Basic access more due to more collision durations. Since most of packets in the network applications are usually small, RTS/CTS access decreases throughput.

Figure 2 shows fairness among different flows in the network. Fairness is defined as the number of flows that can deliver at least one packet over the total number of them. When Basic access is used and packet size is small, more flows can deliver their packets to their destinations. More increase in the 


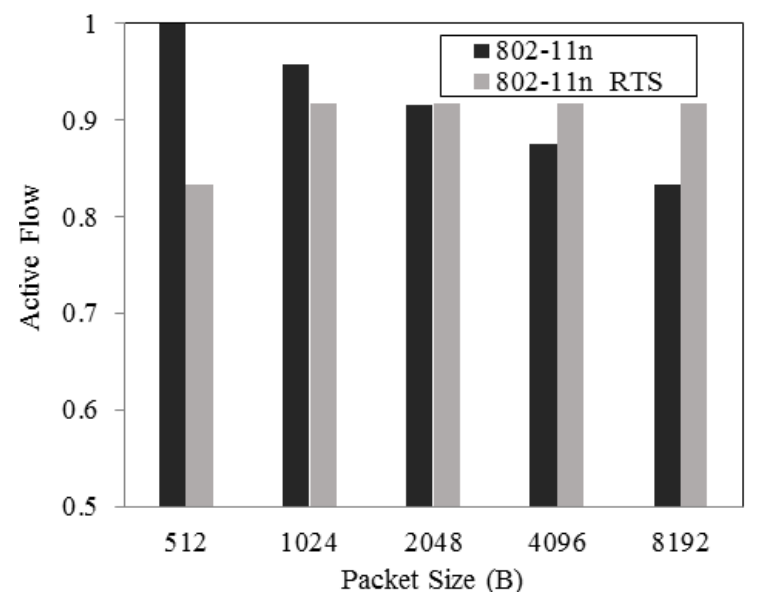

Fig. 2: Fairness among different flows

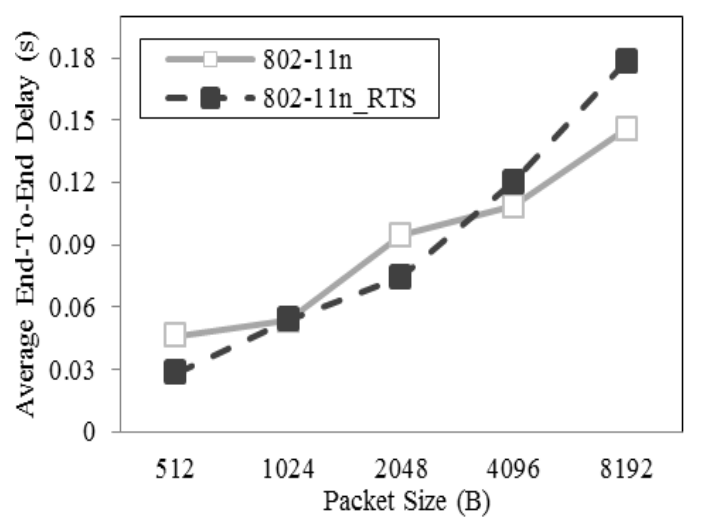

Fig. 3: Average end-to-end delay in basic access and RTS/CTS access

packet size results in loss of fairness, i.e., fewer flows can deliver their packets due to increase in collision durations. Enabling RTS/CTS solves this problem, but it suffers from the same problem for the smaller packet sizes.

Figure 3 shows the average end-to-end packet delay of flows. For small and medium packet sizes, Basic access increases delay, while using RTS/CTS access, large packets experience loner delays.

Figure 4 shows the amount of data, in terms of Mega Bits, that has been dropped along the delivery path. As packet size increases, the network becomes more saturated. RTS/CTS access results in more data packet drops because only RTS packets (which are small) collide and its short retry limit counter reaches to its maximum value sooner. This results in faster packet drops. Basic access, in comparison, holds collided packets for a longer duration and does not drop them.

Packet delivery ratio, the number of delivered data packets over the total number of generated data packets, is shown in Fig. 5. Since RTS/CTS access imposes more control overheads and decreases the

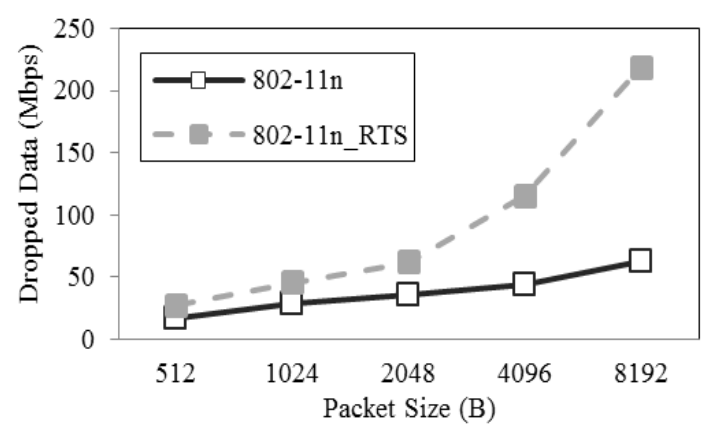

Fig. 4: Dropped data basic access and RTS/CTS access

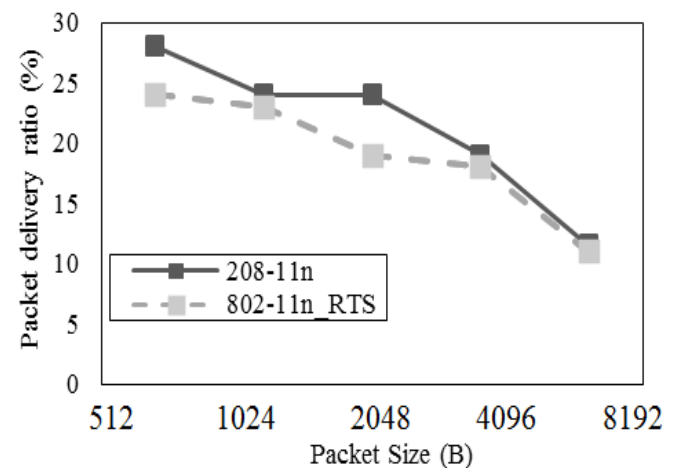

Fig. 5: Packet delivery ratio basic access and RTS/CTS access

MAC throughput, its delivery ratio is lower than that of Basic access.

The above results imply that RTS/CTS access is useful in few situations while it decrease efficiency in most of them, i.e., the RTS threshold is not always dependent to the packet transmit ion time. Since control overheads are crucial in high-speed wireless networks, there is a tradeoff between the two modes.

Although Basic access outperforms RTS/CTS access, eliminating RTS/CTS results in wireless inherent problems, hidden and exposed terminals. Therefore, setting the best RTS threshold is dependent to different packet parameters in the network such as packet size and the experienced delay. By thoroughly investigating the above results, we select the following main parameters affecting RTS threshold which are as follows:

- Packet size

- Hop counts passed so far by a packet (progress)

- Delay experienced by a packet so far

\section{RESULTS AND DISCUSSION}

We propose Routing-Aware Stochastic RTS (RAS-RTS) to set the RTS threshold based on the parameters discussed earlier. RAS-RTS uses Packet size (s), Progress (p) and Delay (d) as input parameters and as a result, an RTS frame is sent with the uniform probability of $F(s, p, d) \in[0,1]$ where, 


$$
F(s, p, d)=\alpha f_{1}(s)+\beta f_{2}(p)+\gamma f_{3}(d)
$$

In Acharya et al., (2008), $\alpha+\beta+\gamma=1$ and $f_{1}(s), f_{2}(p), f_{3}(d) \in[0,1] . f_{1}, f_{2}$, and $f_{3}$ take inputs and show the importance of each parameter in the current packet state in the network. $f_{1}$ is defined as:

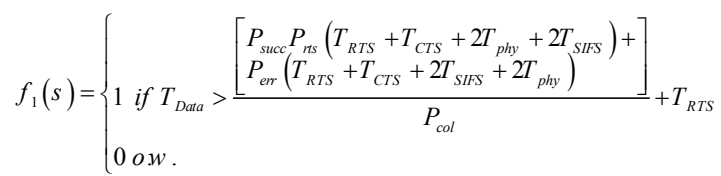

where, $T_{\text {Duta }}, T_{R T S}, T_{C T S}, T_{\text {phy }}, T_{\text {SIISS }}, P_{\text {col }}, P_{\text {succ }}, P_{r s s}$ and $P_{e r}$ are data, RTS, CTS, physical header, SIFS durations, probability of collision, successful, RTS and erroneous transmissions. It is based on results of Tinnirello et al. (2005) and finds the optimal RTS threshold. The other function is given by:

$$
f_{2}(p)=\frac{\text { Current hop count }}{\text { Total hop count }}
$$

which is the percent of the path that a packet has traversed so far.

By $x_{i}=\operatorname{AVG}\left(d_{i, 1}, \ldots, d_{i, t-1}\right)$ we assume that nodes keep the average packet delay of the crossing flow $i$ from the moment 1 to $t-1 . d_{i, t}$ is the experienced delay of the arriving packet of the flow $i$ at time $t$. Therefore:

$$
f_{3}\left(d_{i, t}\right)=\min \left(\frac{d_{i, t}}{x_{i}}, 1\right)
$$

We implemented RAS-RTS in NS-2; simulation parameters are the same as those in the previous Section. Since we need to balance the three functions, we experimentally assigned $0.45,0.30$ and 0.25 to $\alpha, \beta$ and $\gamma$, respectively.

In the following figures, "802.11n" shows results of Basic access and "802.11n_RTS_Base" shows how the protocol behaves if RTS is enabled and "802.11n_RTS_OPT" shows results of applying RAS-RTS.

Figure 6 shows throughput of the three approaches. RAS-RTS increases throughput up to $25 \%$ in comparison with the other approaches. This is due to using appropriate RTS thresholds during packet delivery paths. The decrease observed for large packets is due to more packet drops.

Figure 7 shows fairness of the three approaches among active flows. RAS-RTS has the most fairness among the other approaches.

The average end-to-end delays of the three approaches are illustrated in Fig. 8. RAS-RTS has

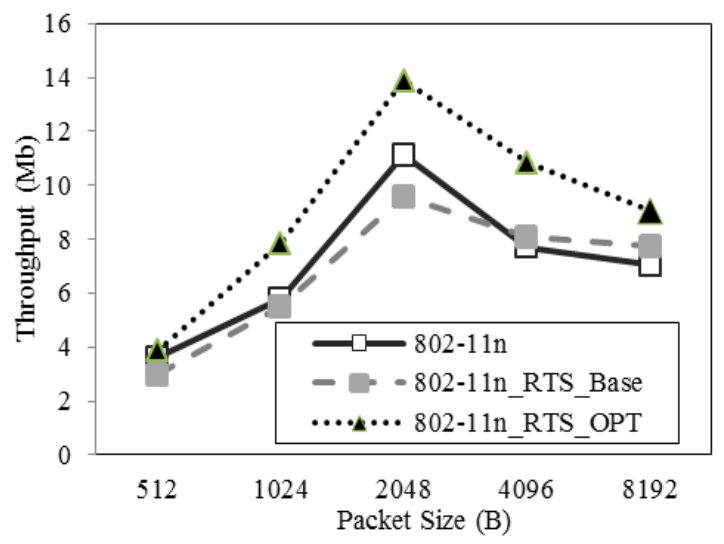

Fig. 6: Throughput vs. various packet sizes in three approaches

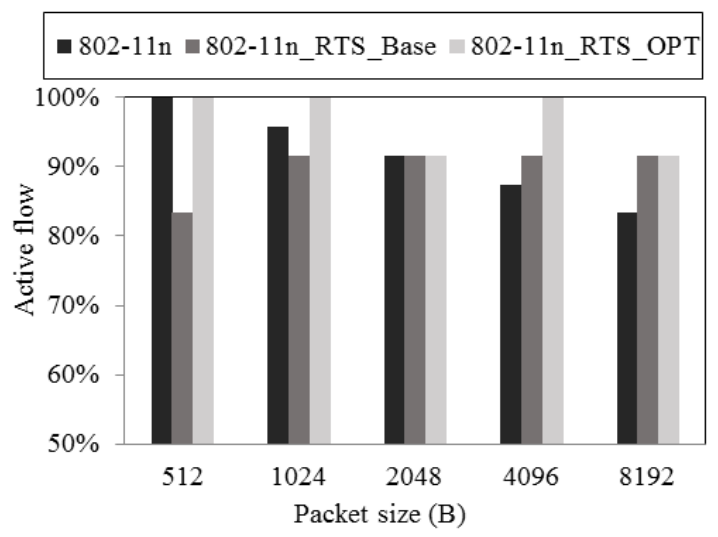

Fig. 7: Fairness among active flows in three approaches

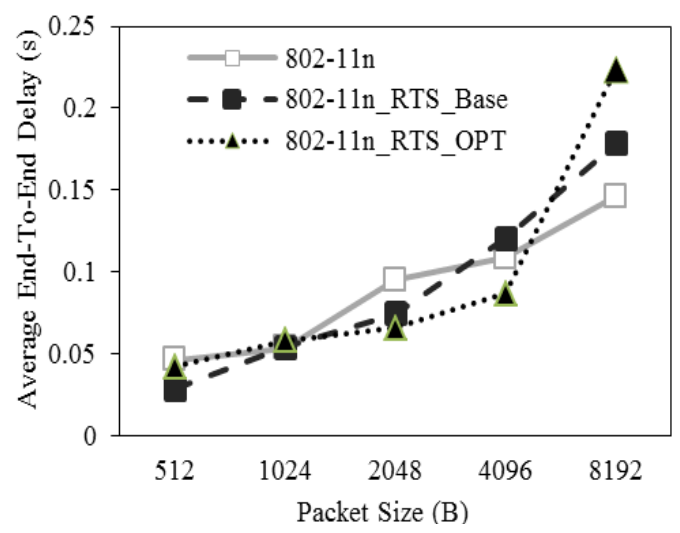

Fig. 8: Average end-to-end delay in three approaches

better delay response in the average especially where the packet size is medium.

Dropped data for three approaches are shown in Fig. 9. Due to more use of RTS/CTS to provide high fairness, it drops more data packets than Basic access.

The main goal of RAS-RTS is to increase delivery ratio and referring to Fig. 10, it improves 


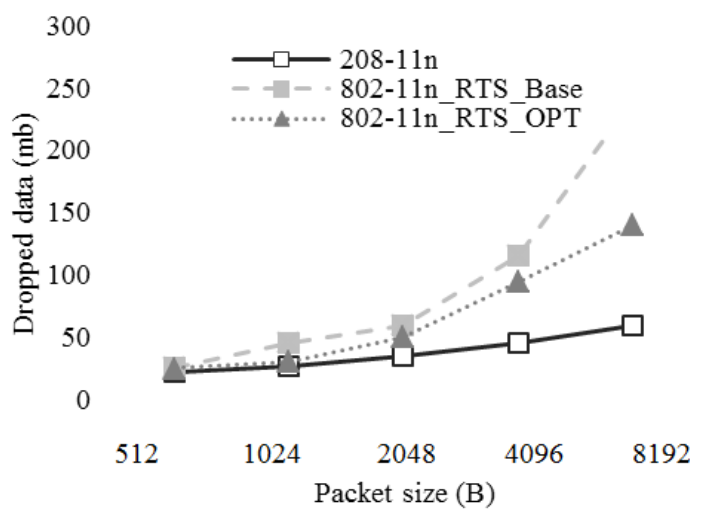

Fig. 9: Dropped data in three approaches

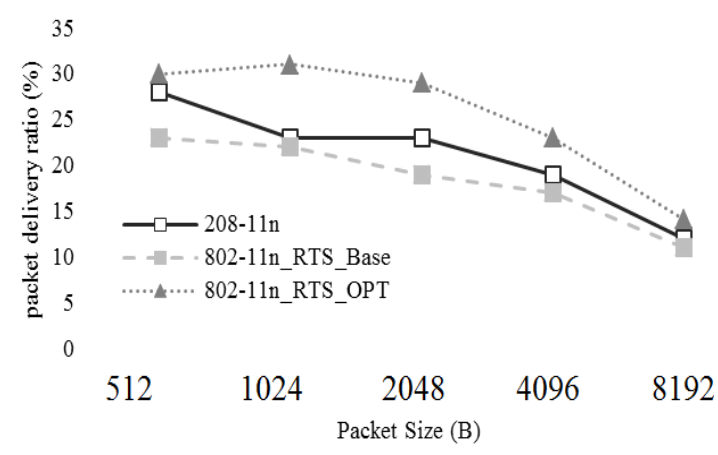

Fig. 10: Packet delivery ratio in three approaches

delivery ratio. The above results show that RAS-RTS is successful in increasing throughput as well as providing high fairness among nodes. It also reduces the end-to-end delay for medium size packets. Since RAS-RTS only uses available parameters at MAC and network layers and only affects RTS threshold, it does not impose any extra overheads to network protocols and node design.

\section{CONCLUSION}

In this study, we introduced a problem which we called RTS threshold problem. Since high-speed wireless ad hoc networks suffer from high control overheads, control packets are usually discarded although it may lead to inherent problems of the wireless environment such as hidden and exposed terminals. We showed that RTS threshold not only depends on the packet size, but it is also dependent to different parameters such as the number of passed hops and delay. We proposed a novel method, RAS-RTS, that decides when to use RTS/CTS access using the aforementioned parameters. Results show that RASRTS improves network efficiency in terms of throughput, delay and delivery ratio. As future works, we will work on more appropriate setting of weighting factors $\alpha, \beta$ and $\gamma$.

\section{REFERENCES}

Acharya, P.A.K., A. Sharma, E.M. Belding, K.C. Almeroth and K. Papagiannaki, 2008. Congestionaware rate adaptation in wireless networks: A measurement-driven approach. Proceeding of the 5th Annual IEEE Communications Society Conference on Sensor, Mesh and Ad Hoc Communications and Networks (SECON'08).

Aghayi, N., 2016. Revenue efficiency measurement with undesirable data in fuzzy DEA. Proceeding of the 7th International Conference on Intelligent Systems, Modelling and Simulation (ISMS), pp: 109-113.

Aghayi, N., 2017. Cost efficiency measurement with fuzzy data in DEA. J. Intell. Fuzzy Syst., 32(1): 409-420.

Aghayi, N. and B. Maleki, 2016. Efficiency measurement of DMUs with undesirable outputs under uncertainty based on the directional distance function: Application on bank industry. Energy, 112: 376-387.

Aghayi, N. and Z. Ghelejbeigi, 2016. Improving cost efficiency by resource allocation. Far East J. Math. Sci., 99(11): 1633-1643.

Aghayi, N., M. Tavana and M.A. Raayatpanah, 2016. Robust efficiency measurement with common set of weights under varying degrees of conservatism and data uncertainty. Eur. J. Ind. Eng., 10(3): 385-405.

Bahrami, M. and M. Effatparvar, 2017. A novel thermal aware routing algorithms for body sensor network. CiiT Int. J. Network. Commun. Eng., 9(4): 81-85.

Banchs, A. and L. Vollero, 2006. Throughput analysis and optimal configuration of 802.11e EDCA. Comput. Networks, 50(11): 1749-1768.

Bianchi, G., 2000. Performance analysis of the IEEE 802.11 distributed coordination function. IEEE J. Sel. Area. Comm., 18(3): 535-547.

Effatparvar, M. and M.S. Garshasbi, 2014. A genetic algorithm for static load balancing in parallel heterogeneous systems. Procedia-Soc. Behav. Sci., 129: 358-364.

Effatparvar, M., M. Dehghan and A.M. Rahmani, 2016. A comprehensive survey of energy-aware routing protocols in wireless body area sensor networks. J. Med. Syst., 40(9): 201.

Effatparvar, M., M. Dehghan and A.M. Rahmani, 2017. Lifetime maximization in wireless body area sensor networks. Biomed. Res., 28(22).

EffatParvar, M., M. Effatparvar, A. Bemana and M. Dehghan, 2007. Determining a central controlling processor with fault tolerant method in distributed system. Proceeding of the 4th International Conference on Information Technology (ITNG'07), pp: 658-663. 
Fazelpour, M., P. Shankar and J.D. Summers, 2016. Developing design guidelines for meso-scaled periodic cellular material structures under shear loading. Proceeding of the ASME 2016 International Design Engineering Technical Conferences and Computers and Information in Engineering Conference, pp: 7.

Frohn, S., S. Gubner and C. Lindemann, 2010. Analyzing the effective throughput in multi-hop IEEE 802.11n networks. Comput. Commun., 34(16): 1912-1921.

Garshasbi, M.S. and M. Effatparvar, 2013. High performance scheduling in parallel heterogeneous multiprocessor systems using evolutionary algorithms. I. J. Int. Intell. Syst. Appl., 5(11): 89-95.

Karlsson, J., A. Kassler and A. Brunstrom, 2009. Impact of packet aggregation on TCP performance in wireless mesh networks. Proceeding of the 5th IEEE WoWMoM Workshop on Hot Topics in Mesh Networking (HotMESH'09), pp: 1-7.

Kim, W., H.K. Wright and S.M. Nettles, 2008. Improving the performance of multi-hop wireless networks using frame aggregation and broadcast for TCP ACKs. Proceeding of the ACM CoNEXT, Madrid, Spain.

Li, T., Q. Ni, D. Malone, D. Leith, Y. Xiao and T. Turletti, 2009. Aggregation with fragment retransmission for very high-speed WLANs. IEEE/ACM T. Networking, 17(2): 591-604.

Lin, Y. and V.W.S. Wong, 2006. WSN01-1: Frame aggregation and optimal frame size adaptation for IEEE 802.11n WLANs. IEEE Globecom, pp: 1-6.

Molaiy, S. and M. Effatparvar, 2014. Scheduling in grid systems using ant colony algorithm. Int. J. Comput. Network Inform. Secur., 6(2): 16-22.

Perkins, C.E. and E.M. Royer, 1999. Ad hoc ondemand distance vector routing. Proceeding of the 2nd IEEE Workshop on Mobile Computing Systems and Applications, New Orleans, LA, pp: 90-100.
Rostamy, M.M. and N. Aghayi, 2011. Measuring overall profit efficiency with fuzzy data. J. Math. Extens., 5(2): 73-90.

Saffar Ardabili, J., N. Aghayi and A. Monzali, 2007. New efficiency using undesirable factors of data envelopment analysis. Adv. Model. Optimiz., 9(2): 249-255.

Salehpour, S. and N. Aghayi, 2015. The most revenue efficiency with price uncertainty. Int. J. Data Envelop. Anal., 3(1): 575-592.

Shankar, P., M. Fazelpour and J.D. Summers, 2015. Comparative study of optimization techniques in sizing mesostructures for use in nonpneumatic tires. J. Comput. Inform. Sci. Eng., 15(4): 041009.

Shrivastava, V., S. Rayanchu, J. Yoonj and S. Banerjee, 2008. 802.11n under the microscope. Proceeding of the 8th ACM SIGCOMM Conference on Internet measurement (IMC'08), pp: 105-110.

Tinnirello, I., Ch Sunghyun and K. Youngsoo, 2005. Revisit of RTS/CTS exchange in high-speed IEEE 802.11 networks. Proceeding of the 6th IEEE International Symposium WoWMoM'05, pp: 240-248.

Wang, C.Y. and H.Y. Wei, 2009. IEEE 802.11n MAC enhancement and performance evaluation. Mob. Networks Appl., 14(6): 760-771.

Yoder, M., Z. Satterfield, M. Fazelpour, J.D. Summers and G. Fadel, 2015. Numerical Methods for the design of meso-structures: A comparative review. Proceeding of the ASME 2015 International Design Engineering Technical Conferences and Computers and Information in Engineering Conference, pp: V02BT03A003-V02BT03A003. 